\title{
Research on Parametric Model for Surface Processing Prediction of Aero-Engine Blades
}

\author{
Lei QIU, Shiming J゙*, Xi ZENG, Dapeng TAN, Man GE
}

\begin{abstract}
This paper presented a method for establishing a blade surface machining prediction model based on a parametric model. The abrasive grain state of the grinding tool was divided into initial wear stage, stable wear stage and sharp wear stage. Based on this, a parametric prediction model of engine blade surface material removal was established. In this paper, the simulation of blade surface machining was carried out. In this work, the blade was divided into several sections according to the direction from the blade root to the blade tip. A certain curve of the outer contour was fitted with a specific arc to reduce the calculation amount. Through a series of simulation calculations, the expressions of the above parametric prediction model were obtained, and several experiments were carried out to verify the feasibility of the prediction model, and the results were analyzed.
\end{abstract}

Keywords: aero engine; blades; parametric model; surface processing prediction

\section{INTRODUCTION}

As the heart of the aircraft, advanced aero-engine is a strategic product related to the development of national military security and civil aviation; it is praised as "the jewel in the crown of modern industrial technology". The blade is a key component of the aero-engine. The design and manufacturing level of the blade largely affects the key technical indicators such as the engine's thrust-to-weight ratio, fuel efficiency, compressor sub-ratio, and service life.

The design technology and processing technology of the engine blades are very complicated. This complexity is mainly reflected in the following aspects. First, the number of blades is large. Take the Russian АЛ-31Ф (for the Su27 combat plane) as an example, there are 13 stages, of which 4 are low pressure compressors and 9 are high pressure compressors. Second, the size of each stage of the blade is different, the minimum blade length is only $20 \mathrm{~mm}$, the maximum blade length is $800 \mathrm{~mm}$, and the third, the geometric shape is complex, the blade profile is highly distorted, and the profile is composed of various complexities surface. Fourth, the blade working environment is harsh, it needs to run under high temperature and high pressure for a long time, the material of the blade is generally difficult to process materials.

Since the 1960s, the large-scale industrialization of aviation products has led to the development of engine blade manufacturing technology, which in turn has improved the overall performance of aircraft engines. Since the compressor blades and turbine blades of the engine are made of difficult-to-machine materials, casting and forging techniques are applied more frequently during the machining process [1-2].

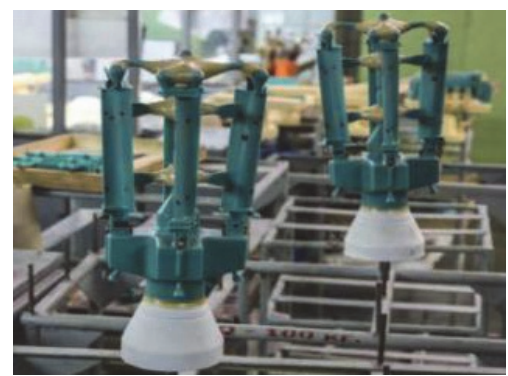

Figure 1 Lost wax method for precision casting of engine blades

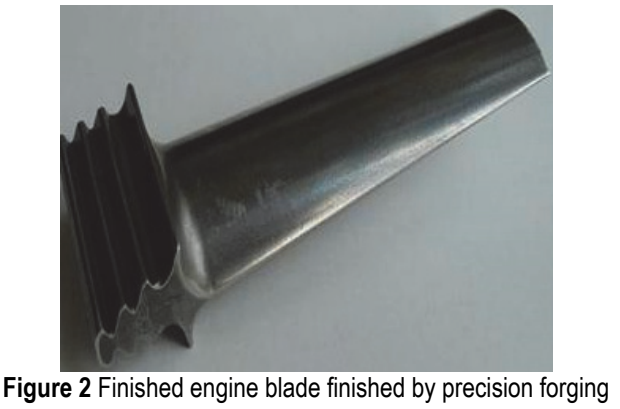

Fig. 1 is a picture of Ufa engine production complex (UMPO) using a lost-wax method for precision casting of engine blades. Fig. 2 is a finished view of an engine blade prepared by precision forging. Precision-casting blades are a casting method with no margin. This is a common blade manufacturing method with mature technology and high efficiency. However, in the manufacture of highperformance engines for fifth-generation fighter aircraft, the shortcomings of this manufacturing method are obvious. The turbine blade with high-temperature alloy as the material, the single crystal solidification technology is still not mature enough, because the solidification direction of the single crystal superalloy liquid is in multiple directions, and the crystal grains will be generated outside the blade root, which is not conducive to further improvement of engine performance. In the process of solidification of metal liquid, the volume of the compressor will shrink more obviously, and the geometrical characteristics of the blade will change. A kind of casting anti-deformation technology to solve this defect is not yet complete mature.

The blade machining allowance made by precision forging technology is very small, which can reach almost $0.3 \mathrm{~mm}$ [3-5], the blade shape accuracy is higher [6], the surface roughness of the blade blank reaches $R a=0.4 \mathrm{um}$, so it is believed that precision forging technology has been applied to most blade manufacturing. However, precision forging also has shortcomings. Blades made by this technology may have potential cracks. This is a fatal flaw for any engine. In addition, the compressors of large bypass ratio aero-engines tend to with the design of the damper table, at the same time, the turbine blades of these highperformance aero-engines will have hollow structures, surface film holes and other structures, which are the 
problems that the current precision forging technology cannot solve. Some small turbojet engines used to drive missile flight, due to its one-time use characteristics, need to greatly reduce manufacturing costs [7], using the above mentioned methods for production, cannot meet this characteristic.

The rapid development of numerical control technology makes it possible to mill complex curved surfaces. For example, Takeuchi Y et al. studied the noninterference processing trajectory generated based on CAM [8], chen-hua she et al. based on $\mathrm{C}++$ Builder and open GL A processing platform for three-axis and two-turn five-axis machine tools [9-10], Loney, Ozsoy et al. proposed an equal parameter method for curved multi-axis CNC machining trajectory [11], Ho S and Sarma $\mathrm{S}$ et al. How to improve the processing efficiency under the premise of ensuring the processing accuracy [12]. Based on a series of researches, some commercial software has been developed, which effectively solve the geometric expression of complex surfaces, tool path generation and other issues, such as Master CAM, Hyper Mill, CATIA and so on. These techniques are also used in the milling of aerospace engine blades.

\section{ENGINE BLADE CNC MILLING METHOD}

The development of CNC multi-axis milling directly drives the development of the aero-engine manufacturing industry, which brings many favorable factors to the engine blade manufacturing [13-15]. Fig. 3 shows an engine blade with a damper table machined by a CNC multi-axis milling machine. It is obvious that the milled blade surface has a strip-like milled texture that is formed by the inconsistent residual height after milling. Fig. 4 is a physical diagram of a CNC milled blade that results in a lack of surface quality consistency and can be identified as a surface defect that does not meet blade design requirements.

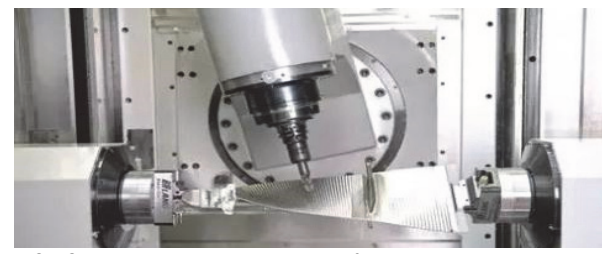

Figure $3 \mathrm{CNC}$ multi-axis milling machine for machining engine blades with damper table

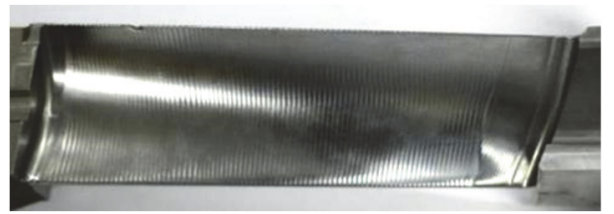

Figure 4 CNC milling forming blade

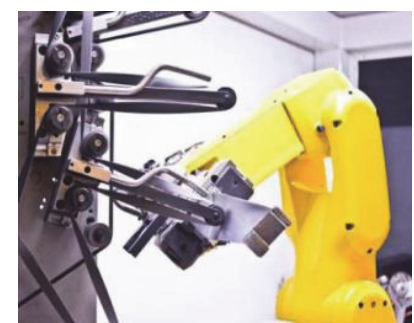

Figure 5 AV\&R Aerospace's engine blade polishing system

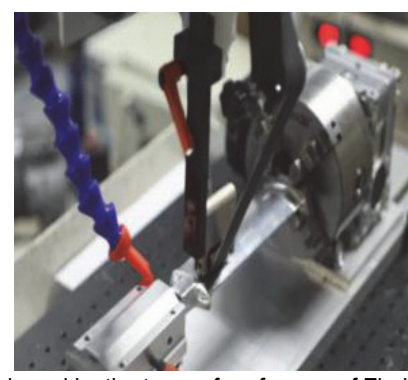

Figure 6 Method used by the team of professors of Zhejiang University of Technology

Therefore, it is necessary to carry out more in-depth processing of the blade surface in order to obtain the surface quality of the engine blade that meets the design requirements after numerical control multi-axis milling. The use of abrasive belts for the polishing of blade surface is a very efficient processing method and can be automated high efficient. Fig. 5 shows the abrasive belt-based engine blade polishing system developed by the famous AV\&R Aerospace Company in Canada. Fig. 6 shows the blade deep processing method used by the team of Professor Ji Shiming from Zhejiang University of Technology in China. Before that, a manual belt machine method was used in most of the manufacturing plants [16].

Fig. 7 is the actual blade obtained by numerical control milling-abrasive belt polishing. This is a compressor blade made of titanium alloy. Because of its chemical properties, it is more active, tough, and has low thermal conductivity. The abrasive particles generated by grinding are not easily separated from the blade surface, and the local temperature rises, which will cause partial damage to the blade surface. Moreover, when the abrasive belt is in contact with the blade, the processed blade will undergo a slight elastic deformation, thereby affecting the material removal accuracy of the blade surface.

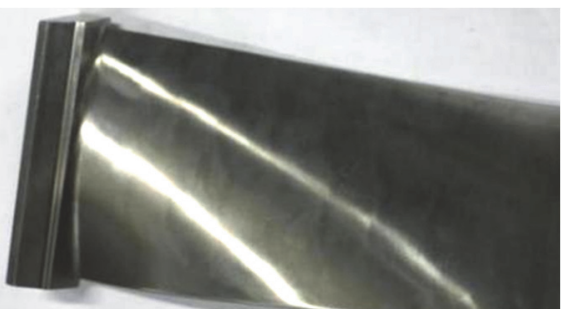

Figure 7 Blade processed by CNC milling-abrasive belt grinding and polishing

In summary, the parametric model of the engine blade under grinding conditions has a very positive significance for the deepening of the aero-engine blade belt for milling.

\section{REMOVAL MECHANISM AND PARAMETRIC MODEL}

In the process of grinding and polishing the surface of the blade, the abrasive belt is always in the process of gradual failure. The main performance is that the grinding amount is gradually reduced and the effective grinding time is increased. We can divide the life cycle of the abrasive belt into three stages according to the amount of grinding. $R_{1}$ represents the surface removal rate of the blade in the initial wear stage of the grinding tool, and $R_{2}$ represents the removal rate of the blade surface in the stable wear stage of the grinding tool. $R_{3}$ represents the removal rate of the blade surface during the severe wear phase of 
the grinding tool, i.e.:

$$
\begin{aligned}
& R_{1}=\frac{R_{\max }}{\sqrt{2 \pi} \sigma} \exp \left[-\frac{(t-\mu)^{2}}{2 \sigma^{2}}\right], 0 \leq t \leq t_{0} \\
& R_{2}=G_{\mathrm{p}} G_{\mathrm{c}} W_{\mathrm{n}}, t_{0} \leq t \leq t_{1} \\
& R_{3}=-a t^{2}+b t+c, t_{1} \leq t \leq t_{\mathrm{rep}}
\end{aligned}
$$

In the above formulas, $G_{\mathrm{p}}$ is the grinding process parameter, $G_{\mathrm{c}}$ is the grinding condition, and $W_{\mathrm{n}}$ is the motion condition parameter. The detailed expressions of these three variables are:

$$
\begin{aligned}
& G_{\mathrm{p}}=K_{1} v_{\mathrm{S}}^{\lambda_{1}} P^{\lambda_{2}} v_{\mathrm{w}}^{\lambda_{3}} F_{\mathrm{t}}^{\lambda_{4}} \\
& G_{c}=K_{2} G_{m}^{\lambda^{5}} H^{\lambda^{6}} C_{c}^{\lambda^{7}} \\
& W_{n}=K_{3} f^{\lambda_{8}} N^{\lambda_{9}}
\end{aligned}
$$

In the above three equations, $K_{1}, K_{2}$, and $K_{3}$ are coefficients for compensating three important parameters of $G_{\mathrm{p}}, G_{\mathrm{c}}$, and $W_{\mathrm{n}}, \lambda_{\mathrm{n}}$ is an index for correcting each parameter, and $\mathrm{P}$ is the pressure at which the abrasive belt is in contact with the blade, $H$ is the hardness of the contact wheel of the belt machine, $C_{\mathrm{c}}$ is the cooling condition constant, $W_{\mathrm{n}}$ is the coefficient indicating the amount of change of the movement state, $f$ is the frequency indicating the circular motion of the belt, and $\mathrm{N}$ is the number of times the belt pulley rotates in contact with the wheel for a certain period of time.

Bring the above equation into the Eq. (2), then:

$$
R_{2}=K_{1} K_{2} K_{3} v_{s}^{\lambda_{1}} P^{\lambda_{2}} v_{w}^{\lambda_{3}} F_{t}^{\lambda_{4}} G M^{\lambda_{5}} H^{\lambda_{6}} C_{c}^{\lambda_{7}} f^{\lambda_{8}} N^{\lambda_{9}}
$$

Let's set it up $K=K_{1} K_{2} K_{3}$, then the above formula becomes:

$$
R_{2}=K v_{s}^{\lambda_{1}} P^{\lambda_{2}} v_{w}^{\lambda_{3}} F_{t}^{\lambda_{4}} G M^{\lambda_{5}} H^{\lambda_{6}} C_{c}^{\lambda_{7}} f^{\lambda_{8}} N^{\lambda_{9}}
$$

Obviously, the working wear and loss of the tool is continuous, then the surface removal rate of the blade surface is also continuous. At the intersection of the initial loss and the stable loss, there must be $R_{1}=R_{2}$, then we can get:

$$
\frac{R_{\max }}{\sqrt{2 \pi} \sigma} \exp \left(\frac{(t-\mu)^{2}}{2 \sigma^{2}}\right)=K v_{s}^{\lambda_{1}} P^{\lambda_{2}} v_{w}^{\lambda_{3}} F_{t}^{\lambda_{4}} G_{m}^{\lambda_{5}} H^{\lambda_{6}} C_{c}^{\lambda_{7}} f^{\lambda_{8}} N^{\lambda_{9}}
$$

we can get the following formula:

$$
R_{\max }=\sqrt{2 \pi} \sigma \cdot \exp \left(\frac{(t-\mu)^{2}}{2 \sigma^{2}}\right) \cdot K v_{s}^{\lambda_{1}} P^{\lambda_{2}} v_{w}^{\lambda_{3}} F_{t}^{\lambda_{4}} G_{m}^{\lambda_{5}} H^{\lambda_{6}} C_{c}^{\lambda_{7}} f^{\lambda_{8}} N^{\lambda_{9}}
$$

In the above formula, $\sigma$ and $\mu$ are parameters related to the maximum grinding time, and it can be considered that $\mu=t_{\max }$ and $\sigma=\xi t_{\max }$, then, the Eq. (10) becomes:

$$
\begin{aligned}
& R_{\max }=\sqrt{2 \pi} \xi t_{\max } \cdot \exp \left(\frac{\left(t-t_{\max }\right)^{2}}{2 \xi t_{\max }^{2}}\right) . \\
& \cdot K v_{s}^{\lambda_{1}} P^{\lambda_{2}} v_{w}^{\lambda_{3}} F_{t}^{\lambda_{4}} G_{m}{ }^{\lambda_{5}} H^{\lambda_{6}} C_{c}^{\lambda_{7}} f^{\lambda_{8}} N^{\lambda_{9}}
\end{aligned}
$$

In general, when the belt removal ability of the workpiece surface is reduced by $2 / 3$ from its maximum removal capacity, we can think that the cutting ability of the abrasive belt has been reduced to the lowest value, and it is necessary to replace the belt. Restore the cutting ability of the belt to its maximum value. Then, the minimum value of the blade surface removal is:

$$
\begin{gathered}
R_{\min }=\frac{1}{3} R_{\max }=\frac{1}{3} \sqrt{2 \pi} \xi \mathrm{t}_{\max } \bullet \exp \left(\frac{\left(t-\mathrm{t}_{\max }\right)^{2}}{2 \xi \mathrm{t}_{\text {max }}^{2}}\right) \bullet \\
K v_{s}^{\lambda_{1}} P^{\lambda_{2}} v_{w}^{\lambda_{3}} F_{t}^{\lambda_{4}} G_{m}^{\lambda_{5}} H^{\lambda_{6}} C_{c}^{\lambda_{7}} f^{\lambda_{8}} N^{\lambda_{9}}
\end{gathered}
$$

If the time $t$ is divided according to the cutting ability of the abrasive belt, it can be divided into several regions with the boundary points of $t_{0}, t_{1}$, and $t_{2}$, respectively, $t_{\max }$ and $t_{\min }$ respectively represent extreme points in different regions, and $t_{1}$ is $R_{2}$. The continuous and guideable points of the two regions of $V_{3}, t_{\text {rep }}$ need to change the time point of the abrasive belt, it is not difficult to find that the time $t$ and the removal rate of the blade surface will satisfy the following relationship:

$$
\left\{\begin{array}{c}
R_{1}=R_{\max }, t=t_{\max } \\
R_{2}=R_{3}, t=t_{1} \\
R_{3}=R_{\min }, t=t_{\min } \\
R_{3}=0, t=t_{\text {rep }}
\end{array}\right.
$$

Substituting the above equations into Eq. (1), Eq. (2) and Eq. (3), we can solve the expressions of the coefficients $a, b$, and $c$, which are respectively expressed as follows:

$$
\begin{aligned}
& a=K v_{s}^{\lambda_{1}} P^{\lambda_{2}} v_{w}^{\lambda_{3}} F_{t}^{\lambda_{4}} G_{m}{ }^{\lambda_{5}} H^{\lambda_{6}} C_{c}^{\lambda_{7}} f^{\lambda_{8}} N^{\lambda_{9}} . \\
& \left.\cdot \frac{1 / 3 t_{\text {rep }}^{4} \sqrt{2 \pi} \xi t_{\max } \exp \left(\frac{t_{0}-t_{\max }}{\sqrt{2} \xi t_{\max }}\right)^{2}\left(t_{1}-t_{\text {rep }}\right)+t_{\text {rep }}^{3}\left(1-t_{\min } t_{\text {rep }}\right)}{\left(t_{1} t_{\text {rep }}^{2}-t_{1}^{2} t_{\text {rep }}\right)\left(t_{\text {rep }}^{2}-t_{\min }^{2}\right)-\left(t_{\min } t_{\text {rep }}^{2}-t_{\min }^{2} t_{\text {rep }}\right)\left(t_{\text {rep }}^{2}-t_{1}^{2}\right)}\right] \\
& b=K v_{s}^{\lambda_{1}} P^{\lambda_{2}} v_{w}^{\lambda_{3}} F_{t}^{\lambda_{4}} G_{m}{ }^{\lambda_{5}} H^{\lambda_{6}} C_{c}^{\lambda_{7}} f^{\lambda_{8}} N^{\lambda_{9}} . \\
& {\left[\frac{t_{\text {rep }}^{2}\left(t_{\text {rep }}^{2}-t_{\min }^{2}\right)-1 / 3\left(t_{\text {rep }}^{2}-t_{1}^{2}\right) t_{\text {rep }}^{4} \sqrt{2 \pi} \xi t_{\max } \exp \left(\frac{t_{0}-t_{\max }}{\sqrt{2} \xi t_{\max }}\right)^{2}}{\left(t_{1} t_{\text {rep }}^{2}-t_{1}^{2} t_{\text {rep }}\right)\left(t_{\text {rep }}^{2}-t_{\min }^{2}\right)-\left(t_{\min } t_{\text {rep }}^{2}-t_{\min }^{2} t_{\text {rep }}\right)\left(t_{\text {rep }}^{2}-t_{1}^{2}\right)}\right]}
\end{aligned}
$$

$$
\begin{aligned}
& c=K v_{s}^{\lambda_{1}} P^{\lambda_{2}} v_{w}^{\lambda_{3}} F_{t}^{\lambda_{4}} G_{m}{ }^{\lambda_{5}} H^{\lambda_{6}} C_{c}^{\lambda_{7}} f^{\lambda_{8}} N^{\lambda_{9}} . \\
& {\left[\frac{1 / 3\left(t_{1} t_{\text {rep }}^{2}-t_{1}^{2} t_{\text {rep }}\right) t_{\text {rep }}^{2} \sqrt{2 \pi} \xi_{\text {max }} \exp \left(\frac{t_{0}-t_{\max }}{\sqrt{2} \xi t_{\max }}\right)^{2}-\left(t_{\min } t_{\text {rep }}^{2}-t_{\min }^{2} t_{\text {rep }}\right)}{\left(t_{1} t_{\text {rep }}^{2}-t_{1}^{2} t_{\text {rep }}\right)\left(t_{\text {rep }}^{2}-t_{\min }^{2}\right)-\left(t_{\min } t_{\text {rep }}^{2}-t_{\min }^{2} t_{\text {rep }}\right)\left(t_{\text {rep }}^{2}-t_{1}^{2}\right)}\right]}
\end{aligned}
$$


For easy understanding, the material removal of the blade surface can be expressed as follows:

$$
Q=Q_{1}+Q_{2}+Q_{3}=\int_{0}^{t_{O}} V_{1} \mathrm{~d} t+\int_{t_{0}}^{t_{1}} V_{2} \mathrm{~d} t+\int_{t_{1}}^{t_{r e p}} V_{3} \mathrm{~d} t
$$

In the above formula, the time and removal rate factors are explicitly included, and the grinding process parameters of the abrasive belt at various periods are implicitly included, and the leading role is the grinding process of the abrasive belt during the stable wear period parameter.

In order to obtain the various parameters in the above mathematical model, it is necessary to study the prediction model of the belt processing ability. Taking the natural logarithm of both sides of the formula $X$, we can get:

$$
\begin{aligned}
& \ln R_{2}=\ln K+\lambda_{1} \ln v_{\mathrm{s}}+\lambda_{2} \ln P+\lambda_{3} \ln v_{\mathrm{w}}+\lambda_{4} \ln F_{\mathrm{t}}+ \\
& +\lambda_{5} \ln G_{\mathrm{m}}+\lambda_{6} \ln H+\lambda_{7} \ln C_{\mathrm{c}}+\lambda_{8} \ln f+\lambda_{9} \ln N
\end{aligned}
$$

In the regression prediction model calculation, if the sample is enough, then for the $i$-th sample, we can make:

$$
\begin{aligned}
& \vec{Y}_{i}=\ln R_{2 i} \\
& \vec{\beta}_{i}=\left[\ln K, \lambda_{1}, \lambda_{2}, \lambda_{3}, \lambda_{4}, \lambda_{5}, \lambda_{6}, \lambda_{7}, \lambda_{8}, \lambda_{9}\right]_{i}^{T} \\
& \vec{X}_{i}=\left[\ln v_{s}, \ln P, \ln v_{w}, \ln F_{t}, \ln G_{m}, \ln H, \ln C_{c}, \ln f, L \ln N\right]_{i}
\end{aligned}
$$

$Y$ can be described by a linear expression of $\beta$ and $X$, namely:

$$
Y=\beta_{0}+\beta_{1} X_{1}+\beta_{2} X_{2}+\beta_{3} X_{3}+, \ldots,+\beta_{k} X_{k}+\sigma
$$

In the above formula, $\sigma$ is a random error. Further, for $Y_{i}$, there are corresponding $X_{1 i}, X_{2 i}, X_{3 i}, \ldots, X_{k i}(i=1,2, \ldots$, $n$ ) and its expression:

$$
\begin{aligned}
& Y_{i}=\beta_{0 i}+\beta_{1 i} X_{1 i}+\beta_{2 i} X_{2 i}+\beta_{3 i} X_{3 i}+ \\
& \ldots,+\beta_{k i} X_{k i}+\sigma_{i}, i=1,2,3, \cdots, n
\end{aligned}
$$

It can be simply expressed as:

$$
\vec{Y}=\vec{X} \vec{\beta}+\vec{\sigma}
$$

In the above formula, the vector of $\boldsymbol{Y}$ associated with the sample observations can be expressed as:

$$
\vec{Y}=\left[Y_{1}, Y_{2}, Y_{3}, \ldots, Y_{n}\right]^{\mathrm{T}}
$$

$\boldsymbol{X}$ is a matrix related to sample observations and can be expressed as:

$$
\boldsymbol{X}=\left|\begin{array}{ccccc}
1 & 1 & 1 & \cdots & 1 \\
X_{1 n} & X_{1(n-1)} & X_{1(n-2)} & \cdots & X_{11} \\
X_{2 n} & X_{1(n-1)} & X_{2(n-2)} & \cdots & X_{21} \\
\vdots & \vdots & \vdots & \vdots & \vdots \\
X_{k n} & X_{k(n-1)} & X_{k(n-2)} & \cdots & X_{k 1}
\end{array}\right|
$$

The other two vectors in Eq. (24) are the parameter vector $\beta_{k+1}$ and the error vector $\sigma_{n}$, respectively, and their expressions are:

$$
\begin{aligned}
& \beta_{k+1}=\left[\beta_{0}, \beta_{1}, \beta_{2}, \cdots, \beta_{k}\right]^{\mathrm{T}} \\
& \sigma_{n}=\left[\sigma_{1}, \sigma_{2}, \sigma_{3}, \cdots, \sigma_{n}\right]^{\mathrm{T}}
\end{aligned}
$$

If $\delta_{i}$ is the deviation between the sample observation value $Y_{i}$ and the fitting function $y=S(x)$ at the $X_{i}$ point, then $\delta_{i}$ can be written as:

$\delta_{i}=S\left(x_{i}\right)-Y_{i}$

There are linear independent function groups $\Psi_{1}(x)$, $\Psi_{2}(x), \Psi_{3}(x), \ldots, \Psi_{\mathrm{k}}(x)$, which can be used to express the fitting function $S(x)$, that is:

$S(x)=\alpha_{0}+\alpha_{1} \Psi_{1}(x)+\alpha_{2} \Psi_{2}(x)+\cdots+\alpha_{k} \Psi_{k}(x)$

Then, it is not difficult to find a fitting function $S(x)$, so that the sum of the squares of the deviations $\delta_{i}$ becomes the minimum value, that is:

$\left\|\delta_{i}\right\|^{2}=\sum_{i=0}^{n} \delta_{i}^{2}=\sum_{i=0}^{n}\left[S\left(x_{\mathrm{i}}\right)-Y_{i}\right]^{2}=\left.\min \sum_{i=0}^{n}\left[S\left(x_{\mathrm{i}}\right)-Y_{i}\right]^{2}\right|_{S(x) \in \Psi}$

Where:

$\Psi=\operatorname{span}\left\{\Psi_{0}(x), \Psi_{1}(x), \Psi_{2}(x), \ldots, \Psi_{\mathrm{k}}(x)\right\}$

Substituting the Eq. (30) into the Eq. (29):

$$
\begin{aligned}
\left\|\delta_{i}\right\|^{2} & =\sum_{i=0}^{n} \delta_{i}^{2}=\sum_{i=0}^{n}\left[S\left(x_{i}\right)-Y_{i}\right]^{2} \\
& =\sum_{i=0}^{n}\left[Y_{i}-\alpha_{0}-\alpha_{1} \Psi_{1}\left(x_{i}\right)-\alpha_{2} \Psi_{2}\left(x_{i}\right)-, \ldots,-\alpha_{k} \Psi_{k}\left(x_{i}\right)\right]^{2}
\end{aligned}
$$

Find the first-order partial derivatives of $\alpha_{0}, \alpha_{1}, \alpha_{2}, \ldots$, $\alpha_{k}$ in the above equation and make them equal to 0 to obtain their extreme points. The following equations can be obtained:

$$
\left\{\begin{array}{c}
\frac{\partial \delta}{\partial \alpha_{0}}=2 \sum(-1)\left(Y_{i}-\alpha_{0}-\alpha_{1} \Psi_{1}(x)-\alpha_{2} \Psi_{2}(x)-\cdots-\alpha_{k} \Psi_{k}(x)\right)=0 \\
\frac{\partial \delta}{\partial \alpha_{1}}=2 \sum\left(-\Psi_{1}(x)\right)\left(Y_{i}-\alpha_{0}-\alpha_{1} \Psi_{1}(x)-\alpha_{2} \Psi_{2}(x)-, \ldots,-\alpha_{k} \Psi_{k}(x)\right)=0 \\
\frac{\partial \delta}{\partial \alpha_{2}}=2 \sum\left(-\Psi_{2}(x)\right)\left(Y_{i}-\alpha_{0}-\alpha_{1} \Psi_{1}(x)-\alpha_{2} \Psi_{2}(x)-, \ldots,-\alpha_{k} \Psi_{k}(x)\right)=0 \\
\vdots \\
\frac{\partial \delta}{\partial \alpha_{k}}=2 \sum\left(-\Psi_{k}(x)\right)\left(Y_{i}-\alpha_{0}-\alpha_{1} \Psi_{1}(x)-\alpha_{2} \Psi_{2}(x)-, \ldots,-\alpha_{k} \Psi_{k}(x)\right)=0
\end{array}\right.
$$


Simplify the above equations to the following equations:

$$
\left\{\begin{array}{c}
n \alpha_{0}+\alpha_{1} \sum \Psi_{1}(x)+\alpha_{2} \sum \Psi_{2}(x)+, \ldots,+\alpha_{k} \sum \Psi_{k}(x)=\sum Y_{i} \\
\Psi_{1}(x)\left[\alpha_{0}+\alpha_{1} \sum \Psi_{1}(x)+\alpha_{2} \sum \Psi_{2}(x)+, \ldots,+\alpha_{k} \sum \Psi_{k}(x)\right]=\sum \Psi_{1}(x) Y_{i} \\
\Psi_{2}(x)\left[\alpha_{0}+\alpha_{1} \sum \Psi_{1}(x)+\alpha_{2} \sum \Psi_{2}(x)+, \ldots,+\alpha_{k} \sum \Psi_{k}(x)\right]=\sum \Psi_{2}(x) Y_{i} \\
\vdots \\
\Psi_{k}(x)\left[\alpha_{0}+\alpha_{1} \sum \Psi_{1}(x)+\alpha_{2} \sum \Psi_{2}(x)+, \ldots,+\alpha_{k} \sum \Psi_{k}(x)\right]=\sum \Psi_{k}(x) Y_{i}
\end{array}\right.
$$

Then rewritten into a matrix form, the following equations are obtained:

$$
\begin{aligned}
& {\left[\begin{array}{ccccc}
n & \sum \Psi_{1}(x) & \sum \Psi_{2}(x) & \cdots & \sum \Psi_{k}(x) \\
\sum \Psi_{1}(x) & \sum \Psi_{1}^{2}(x) & \sum \Psi_{1}(x) \sum \Psi_{2}(x) & \cdots & \sum \Psi_{1}(x) \sum \Psi_{k}(x) \\
\sum \Psi_{2}(x) & \sum \Psi_{2}(x) \sum \Psi_{1}(x) & \sum \Psi_{2}^{2}(x) & \cdots & \sum \Psi_{2}(x) \sum \Psi_{k}(x) \\
\vdots & \vdots & \vdots & \vdots & \vdots \\
\sum \Psi_{k}(x) & \sum \Psi_{k}(x) \sum \Psi_{1}(x) & \sum \Psi_{k}(x) \sum \Psi_{2}(x) & \vdots & \sum \Psi_{k}^{2}(x)
\end{array}\right] .} \\
& \cdot\left[\begin{array}{c}
\alpha_{0} \\
\alpha_{1} \\
\alpha_{2} \\
\vdots \\
\alpha_{k}
\end{array}\right]=\left[\begin{array}{c}
\sum Y_{i} \\
\sum \Psi_{1}(x) Y_{i} \\
\sum \Psi_{2}(x) Y_{i} \\
\vdots \\
\sum \Psi_{k}(x) Y_{i}
\end{array}\right]
\end{aligned}
$$

We notice that the first matrix on the left side of the above formula can be rewritten into the following form:

$$
\begin{aligned}
& {\left[\begin{array}{ccccc}
n & \sum \Psi_{1}(x) & \sum \Psi_{2}(x) & \cdots & \sum \Psi_{k}(x) \\
\sum \Psi_{1}(x) & \sum \Psi_{1}^{2}(x) & \sum \Psi_{1}(x) \sum \Psi_{2}(x) & \cdots & \sum \Psi_{1}(x) \sum \Psi_{k}(x) \\
\sum \Psi_{2}(x) & \sum \Psi_{2}(x) \sum \Psi_{1}(x) & \sum \Psi_{2}^{2}(x) & \cdots & \sum \Psi_{2}(x) \sum \Psi_{k}(x) \\
\vdots & \vdots & \vdots & \vdots & \vdots \\
\sum \Psi_{k}(x) & \sum \Psi_{k}(x) \sum \Psi_{1}(x) & \sum \Psi_{k}(x) \sum \Psi_{2}(x) & \vdots & \sum \Psi_{k}^{2}(x)
\end{array}\right]=} \\
& =\left[\begin{array}{ccccc}
1 & 1 & 1 & \cdots & 1 \\
x_{11} & x_{12} & x_{13} & \cdots & x_{1 n} \\
x_{21} & x_{22} & x_{23} & \cdots & x_{2 n} \\
\vdots & \vdots & \vdots & \vdots & \vdots \\
x_{k 1} & x_{k 2} & x_{k 3} & \cdots & x_{n k}
\end{array}\right]\left[\begin{array}{ccccc}
1 & x_{11} & x_{21} & \cdots & x_{k 1} \\
1 & x_{12} & x_{22} & \cdots & x_{k 2} \\
1 & x_{13} & x_{23} & \cdots & x_{k 3} \\
\vdots & \vdots & \vdots & \vdots & \vdots \\
1 & x_{1 n} & x_{2 n} & \cdots & x_{n k}
\end{array}\right]=X X^{T}
\end{aligned}
$$

And because:

$$
\left[\begin{array}{c}
\sum Y_{i} \\
\sum \Psi_{1}(x) Y_{i} \\
\sum \Psi_{2}(x) Y_{i} \\
\vdots \\
\sum \Psi_{k}(x) Y_{i}
\end{array}\right]=\left[\begin{array}{ccccc}
1 & 1 & 1 & \cdots & 1 \\
x_{11} & x_{13} & x_{13} & \cdots & x_{1 n} \\
x_{21} & x_{22} & x_{23} & \cdots & x_{2 n} \\
\vdots & \vdots & \vdots & \vdots & \vdots \\
x_{k 1} & x_{k 2} & x_{k 3} & \cdots & x_{k n}
\end{array}\right]\left[\begin{array}{c}
\mathrm{Y}_{1} \\
\mathrm{Y}_{2} \\
\mathrm{Y}_{3} \\
\vdots \\
\mathrm{Y}_{\mathrm{n}}
\end{array}\right]=X \mathrm{Y}
$$

Then, combining Eq. (35) and Eq. (36), the following expression is obtained:

$$
X X^{\mathrm{T}}\left[\begin{array}{c}
\alpha_{0} \\
\alpha_{1} \\
\alpha_{2} \\
\vdots \\
\alpha_{k}
\end{array}\right]=X Y
$$

The expression $\left[\alpha_{0}, \alpha_{1}, \alpha_{2}, \ldots, \alpha_{k}\right]^{\mathrm{T}}$ can be obtained by left multiplying $\left(X X^{\mathrm{T}}\right)^{-1}$ both sides of the above equation, that is: $\left[\begin{array}{c}\alpha_{0} \\ \alpha_{1} \\ \alpha_{2} \\ \vdots \\ \alpha_{k}\end{array}\right]=\left(X X^{\mathrm{T}}\right)^{-1} X Y$

We have noticed:

$$
\begin{aligned}
\left\|\delta_{i}\right\|^{2} & =\sum_{i=0}^{n} \delta_{i}^{2}=\sum_{i=0}^{n}\left[S\left(x_{i}\right)-Y_{i}\right]^{2} \\
& =\sum_{i=0}^{n}\left[Y_{i}-\alpha_{0}-\alpha_{1} \Psi_{1}\left(x_{i}\right)-\alpha_{2} \Psi_{2}\left(x_{i}\right)-, \ldots,-\alpha_{k} \Psi_{k}\left(x_{i}\right)\right]^{2}
\end{aligned}
$$

When $\delta_{i}$ tends to 0 :

$$
Y_{i}=\alpha_{0}+\alpha_{1} \Psi_{1}\left(x_{i}\right)+\alpha_{2} \Psi_{2}\left(x_{i}\right)+, \ldots,+\alpha_{k} \Psi_{k}\left(x_{i}\right)
$$

At this point, as long as the order $X_{i}=\Psi_{i}\left(x_{i}\right)$ it is, then we can get $\left[\beta_{0}, \beta_{1}, \beta_{2}, \ldots, \beta_{k}\right]^{\mathrm{T}}=\left[\alpha_{0}, \alpha_{1}, \alpha_{2}, \ldots, \alpha_{k}\right]^{\mathrm{T}}$, specifically expressed as:

$\left[\begin{array}{c}\beta_{0} \\ \beta_{1} \\ \beta_{2} \\ \vdots \\ \beta_{k}\end{array}\right]=\left(X X^{\mathrm{T}}\right)^{-1} X Y$

It can be seen that whether it is computer simulation or experimental test, as long as the values of $X$ and $Y$ are obtained in advance, the values that $\vec{\beta}_{i}=\left[\ln K, \lambda_{1}, \lambda_{2}, \lambda_{3}, \lambda_{4}, \lambda_{5}, \lambda_{6}, \lambda_{7}, \lambda_{8}, \lambda_{9}\right]_{i}^{\mathrm{T}}$ can be obtained, for the $\boldsymbol{X}$ and $\boldsymbol{Y}$ data obtained by different methods, will get different categories of values, and will also form different Parametric model.

\section{MODEL AND SIMULATION}

Using some advanced simulation software to simulate the machining process, it cannot only visually display the blade surface machining process including macroscopic and microscopic scales, but also obtain a series of parameters including surface material removal, workpiece deformation, temperature, etc. Taking the abrasive belt processing blade as an example, the simulation calculation is carried out in accordance with the objective conditions. As shown in Fig. 8, the schematic diagram of the blade processing is used. For the different curvature parts of the blade, the contact wheels of different diameters are used for the touch.

The shape of modern advanced aero-engine blades is very complicated. In order to reduce the number of compressor stages, small aspect ratio blades are often used. The new form of small aspect ratio blades has a sweep angle. In actual machining, the blades are radially oriented according to the height. Discrete, as shown in Fig. 9b, the 
section of the blade of each section can be seen as two continuous complex curves, which form the suction and pressure surfaces of the blade section.

According to the discrete rule described above, selecting a small number of discrete segments on the blade as the research object can reduce the number of complicated and huge operations, and can also compare and analyze different calculation results of various parts of the blade. At the same time, a section of the belt with the outer contour of the contact wheel is selected as a grinding tool, and a study of the range of the revolution cycle time can eliminate many interference factors. However, for the simulation of surface topography changes, the calculation amount of the computer is still huge. Therefore, the general research is mostly a single abrasive grain or a single cutter head, and even the local part of the cutter can process the blade. Research on simulation calculations based on actual processing conditions is rarely reported.

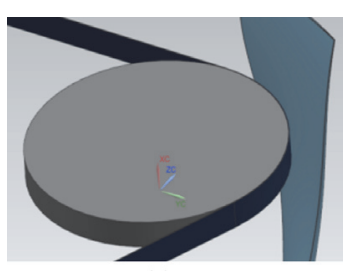

(a)

Figure 8 Positional relationship between contact wheel and blade

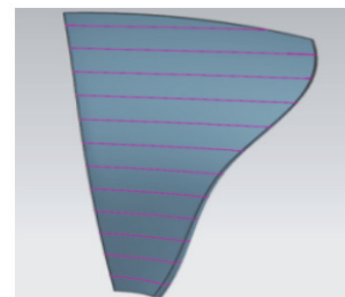

(a)

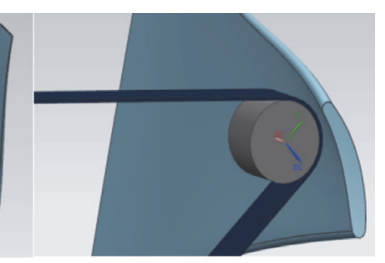

(b)

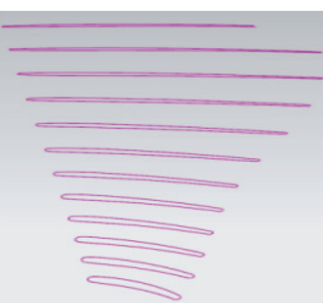

(b)
Figure 9 Discrete curve of shape and contour of blade surface

Fig. 10 shows a typical model in the simulation of this article. The tool here is a length of abrasive belt attached to the contact wheel, but not limited to this belt, it can be other tools. The principle of selecting the length of the belt is that the integral length of the arc length generated when the belt and the contact wheel rotate through an angle $\varphi$ is exactly equal to the relative displacement length of the blade and the contact wheel during this time. Of course, this relative displacement is curved, that is:

$$
\begin{aligned}
\lambda \omega t= & \sum_{i=1}^{j} \int_{x_{1}}^{x_{2}} f_{k 1}(x) \mathrm{d} x+\int_{x_{2}}^{x_{3}} f_{k 2}(x) \mathrm{d} x+ \\
& \int_{x_{3}}^{x_{4}} f_{k 3}(x) \mathrm{d} x+, \ldots,+\int_{x_{n}}^{x_{(n+1)}} f_{k n}(x) \mathrm{d} x
\end{aligned}
$$

Following the three-stage principle of abrasive wear, three grinding tools as shown in Fig. 11 were designed. Taking the belt processing as an example, $a$ is a new belt that has just been activated, and $b$ is a belt that enters a stable wear stage. $c$ is the abrasive belt that enters the stage of intense wear. For these shapes of abrasive particles, simulations were performed under several sets of different

processing conditions.

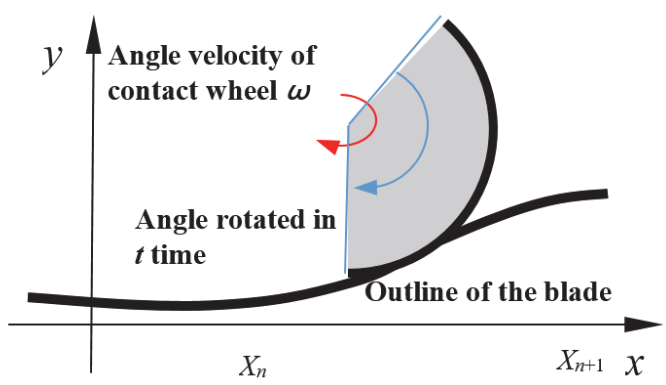

Figure $10 \mathrm{~A}$ typical contour model in simulation

The mesh model of the finite element calculation is shown in Fig. 12. As mentioned above, on a blade section, when the area near the processing area of the blade surface is not too large, the outer contour of the blade can be approximated as a circular arc, the shape and running trajectory of the grinding tool are also approximate arcs, and the motion relationship between the two geometries 1 has been noted in the figure.

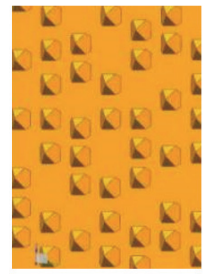

(a) New abrasive

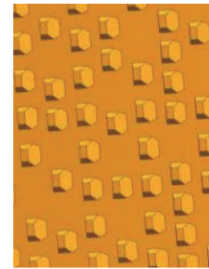

(b) Abrasive grain in stable wear stage

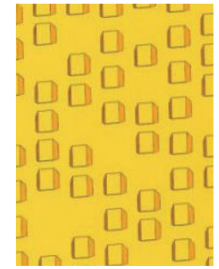

(c) Abrasive grain in the intense wear stage Figure 11 A typical contour model in simulation

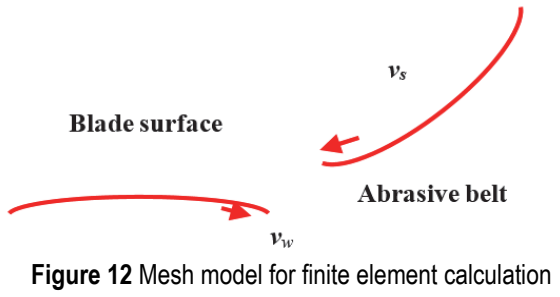

Except for the setting of the abrasive material parameter Gm, which is controlled by experience, other detailed parameter settings can be performed as follows. By changing the parameters of the material, controlling the hardness parameter $H$ of the abrasive grain group, and changing the speed of the reference point rotation (load boundary condition) by setting the speed value of six degrees of freedom in the boundary conditions, thereby controlling the movement frequency f of the abrasive grain group. On this basis, by changing the rotating gear of the abrasive particle group (that is, the distance between the reference point and the abrasive particle group), the linear velocity of the abrasive particle group is controlled; similarly, the same applies to the movement parameter $\mathrm{v}_{\mathrm{w}}$ of the blade surface model. Method: by controlling the MPC contact option parameter (Interaction-MPC Constraint) in the constraint option, control the normal force $\mathrm{P}$ between the blade and the abrasive particle group; by setting the mechanical tangential contact option parameter in the interaction properties (Interaction Property -Tangential Behavior), to control the tangential 
force Ft between the blade and the abrasive grain group; by setting the Step-Time Period, control the number $N$ of rotations of the abrasive grain group within the switch. The cooling condition constant adopts an alternative setting, that is, the reduced air flow rate (the unit is $\mathrm{kg} /$ minute) generated by the high-speed rotation of the grinding tool, so that the workpiece can be effectively cooled during processing.

The results below provide a visual representation of blade surface removal and blade surface topography for different stages of the grinding tool. Fig. 13 is a simulated calculation of the blade surface under a certain set of simulation conditions when the abrasive belt is in the initial wear stage (when the abrasive belt shape is as shown in Fig. 11a). It can be seen from the results that the long strip-like texture produced by milling has been removed, and the machined surface produces a uniform distribution of peakto-valley shapes, which is the surface roughness produced after simulation under this set of conditions. Since our simulation calculations are on a mesoscale scale, the shape of the surface roughness is very obvious, and the average surface removal is about $44.9 \mu \mathrm{m}$ by measuring the blade surface removal depth in proportion.

Fig. 14 is a blade surface obtained by machining a belt under a set of simulated conditions when the belt is in a stable wear stage (the belt abrasive grain shape is as shown in Fig. 11b). Much like the simulation results above, the milled surface of the blade surface is removed and replaced by a new surface roughness resulting from grinding. The surface roughness obtained during the stable wear phase of the belt is less than the initial surface roughness. The surface roughness during the abrasive stable wear stage is higher, but the amount of processing removal is less than the former, about $21.2 \mu \mathrm{m}$.

Fig. 15 is the surface of the blade obtained by simulated machining under a certain set of conditions when the abrasive belt is in a sharp wear stage (the abrasive grain shape at this time is as shown in Fig. 11c). In contrast to the above two sets of simulations, it was found that the amount of surface removal at this time was the smallest, about $14.7 \mu \mathrm{m}$.

Due to the large number of simulation results, we have selected a number of representative results above to demonstrate the grinding effect and surface topography after processing. As mentioned in Chapter 2, the wear of the grinding tool is continuous. Then, in these three stages, we may wish to focus on the second stage, the stable wear stage, for the parameterization model proposed in Chapter 2 , we need to determine a series of parameters in Tab. 1.

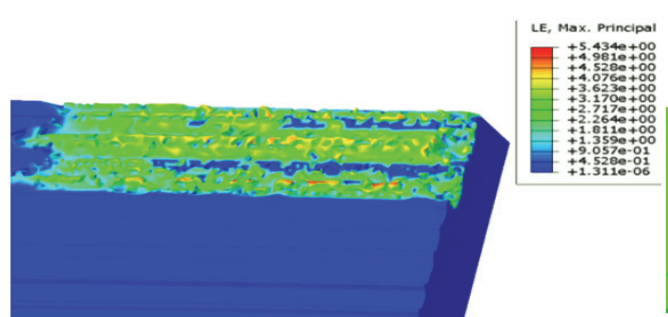

(a)

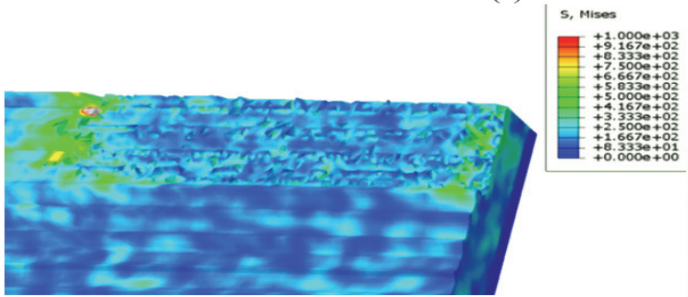

(c)

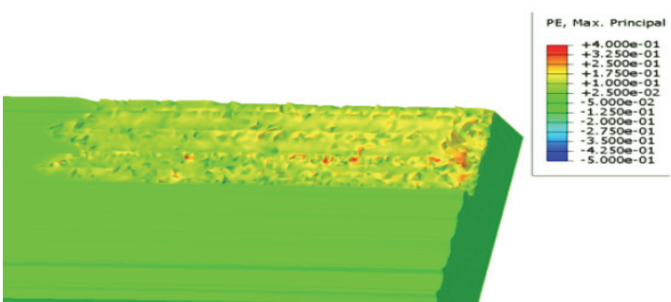

(b)

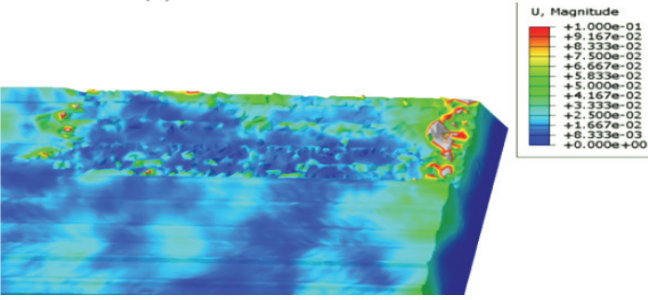

(d)

Figure 13 Simulated calculated blade surface when the abrasive belt is in the initial wear stage

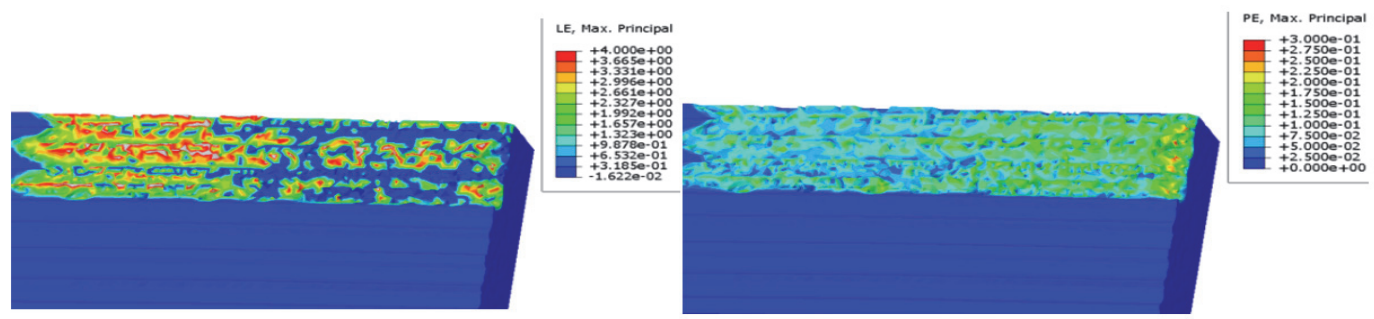

(a)

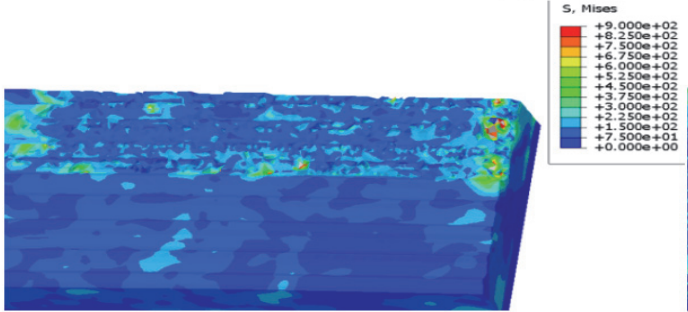

(c) (b)

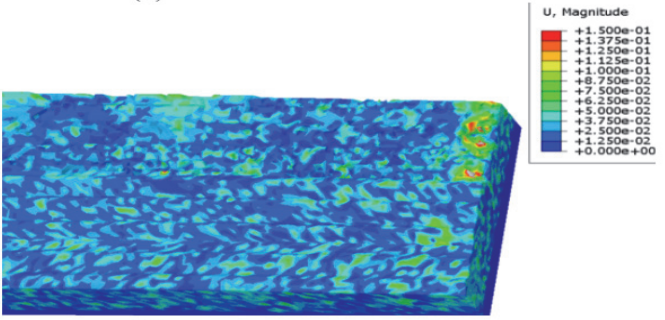

(d)

Figure 14 Simulated calculated blade surface when the abrasive belt is in the stable wear stage 


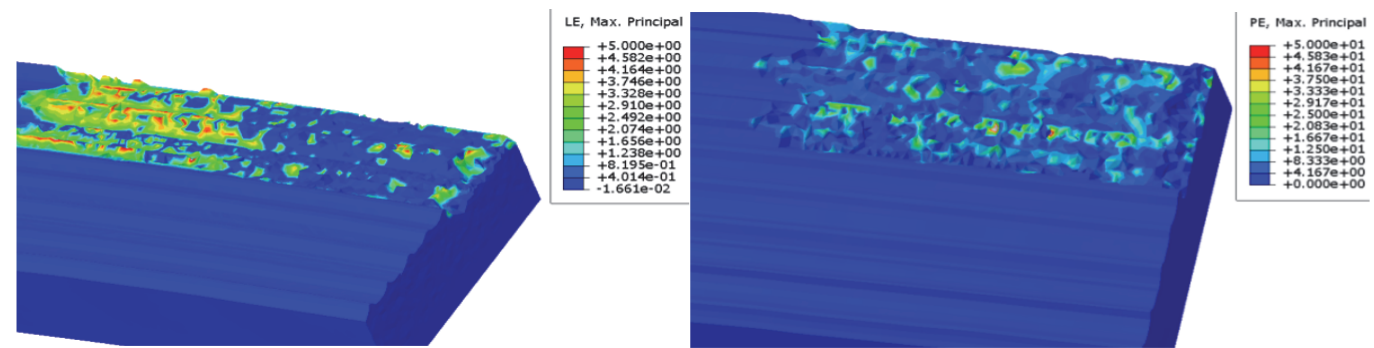

(a)

(b)

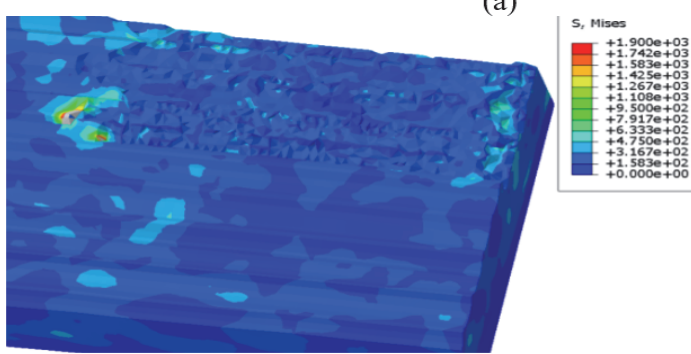

(c)

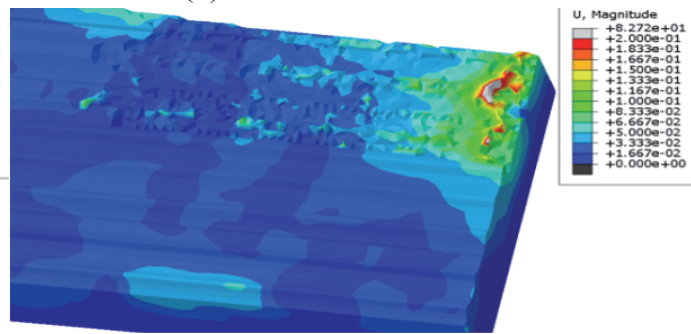

(d)

Figure 15 Simulated calculated blade surface when the abrasive belt is in the intense wear stage

Table 1 Simulation factor table

\begin{tabular}{|c|c|c|c|c|c|c|c|c|c|}
\hline Factor \& team & $\mathrm{v}_{\mathrm{s}}(\mathrm{m} / \mathrm{s})$ & $\mathrm{P}(\mathrm{N})$ & $\mathrm{V}_{\mathrm{w}}(\mathrm{m} / \mathrm{min})$ & $\mathrm{F}_{\mathrm{t}}(\mathrm{N})$ & $\mathrm{G}_{\mathrm{m}}$ & $\mathrm{H}(\mathrm{HA})$ & $\mathrm{C}_{\mathrm{c}}(\mathrm{kg} / \mathrm{min})$ & $\mathrm{N}$ & $\mathrm{f}$ \\
\hline 1 & 4.0 & 2.0 & 0.3 & 0.1 & 1.1 & 15.0 & 1.0 & 10 & 1 \\
\hline 2 & 8.0 & 4.0 & 0.5 & 0.2 & 1.2 & 20.0 & 2.0 & 20 & 2 \\
\hline 3 & 12.0 & 6.0 & 0.7 & 0.3 & 1.5 & 25.0 & 3.0 & 30 & 4 \\
\hline 4 & 16.0 & 8.0 & 0.9 & 0.4 & 1.6 & 30.0 & 4.0 & 40 & 8 \\
\hline
\end{tabular}

This paper selects the conditions in the above table and performs 15 sets of simulation calculations, due to:

$$
\overrightarrow{X_{i}}=\left[\ln v_{\mathrm{s}}, \ln P, \ln v_{\mathrm{w}}, \ln F_{\mathrm{t}}, \ln G_{m}, \ln H, \ln C_{\mathrm{c}}, \ln f, \ln N\right]_{i}
$$

$$
\vec{Y}_{i}=\ln R_{2 i}
$$

Then, the matching method and results of the conditions can be as shown in the following table.

Table 2 Simulation conditions and result data

\begin{tabular}{|c|c|c|c|c|c|c|c|c|c|c|}
\hline & $X_{1 i}$ & $X_{2 i}$ & $X_{3 i}$ & $X_{4 i}$ & $X_{5 i}$ & $X_{6 i}$ & $X_{7 i}$ & $X_{8 i}$ & $X_{9 i}$ & $Y_{i}$ \\
\hline 1 & $1 ; 1.386$ & $1 ; 0.693$ & $1 ;-1.204$ & $1 ;-2.303$ & $1 ; 0.095$ & $1 ; 2.708$ & $1 ; 0$ & $1 ; 2.303$ & $1 ; 0$ & -4.219 \\
\hline 2 & $2 ; 2.079$ & $2 ; 1.386$ & $2 ;-0.693$ & $2 ;-1.609$ & $2 ; 0.182$ & $2 ; 2.996$ & $2 ; 0.693$ & $2 ; 2.996$ & $2 ; 0.693$ & -4.021 \\
\hline 3 & $3 ; 2.484$ & $3 ; 1.792$ & $3 ;-0.357$ & $3 ;-1.204$ & $3 ; 0.405$ & $3 ; 3.219$ & $3 ; 1.099$ & $3 ; 3.401$ & $3 ; 1.386$ & -3.451 \\
\hline 4 & $4 ; 2.773$ & $4 ; 2.079$ & $4 ;-0.105$ & $4 ;-0.916$ & $4 ; 0.470$ & $4 ; 3.401$ & $4 ; 1.386$ & $4 ; 3.689$ & $4 ; 2.079$ & -3.102 \\
\hline 5 & $1 ; 1.386$ & $1 ; 0.693$ & $2 ;-0.693$ & $2 ;-1.609$ & $3 ; 0.405$ & $3 ; 3.219$ & $4 ; 1.386$ & $4 ; 3.689$ & $4 ; 2.079$ & -3.541 \\
\hline 6 & $2 ; 2.079$ & $2 ; 1.386$ & $3 ;-0.357$ & $3 ;-1.204$ & $4 ; 0.470$ & $4 ; 3.401$ & $1 ; 0$ & $1 ; 2.303$ & $1 ; 0$ & -3.668 \\
\hline 7 & $3 ; 2.484$ & $3 ; 1.792$ & $4 ;-0.105$ & $4 ;-0.916$ & $1 ; 0.095$ & $1 ; 2.708$ & $2 ; 0.693$ & $2 ; 2.996$ & $2 ; 0.693$ & -3.741 \\
\hline 8 & $4 ; 2.773$ & $4 ; 2.079$ & $1 ;-1.204$ & $1 ;-2.303$ & $2 ; 0.182$ & $2 ; 2.996$ & $3 ; 1.099$ & $3 ; 3.401$ & $3 ; 1.386$ & -3.433 \\
\hline 9 & $1 ; 1.386$ & $2 ; 1.386$ & $3 ;-0.357$ & $4 ;-0.916$ & $1 ; 0.095$ & $2 ; 2.996$ & $3 ; 1.099$ & $4 ; 3.689$ & $1 ; 0$ & -3.012 \\
\hline 10 & $4 ; 2.773$ & $2 ; 1.386$ & $2 ;-0.693$ & $1 ;-2.303$ & $4 ; 0.470$ & $3 ; 3.219$ & $2 ; 0.693$ & $1 ; 2.303$ & $4 ; 2.079$ & -3.845 \\
\hline 11 & $1 ; 1.386$ & $3 ; 1.792$ & $4 ;-0.105$ & $2 ;-1.609$ & $1 ; 0.095$ & $3 ; 3.219$ & $4 ; 1.386$ & $2 ; 2.996$ & $1 ; 0$ & -3.803 \\
\hline 12 & $2 ; 2.079$ & $1 ; 0.693$ & $4 ;-0.105$ & $3 ;-1.609$ & $2 ; 0.182$ & $1 ; 2.708$ & $4 ; 1.386$ & $3 ; 3.401$ & $1 ; 0$ & -3.752 \\
\hline 13 & $3 ; 2.484$ & $2 ; 1.386$ & $1 ;-1.204$ & $4 ;-0.916$ & $3 ; 0.405$ & $2 ; 2.996$ & $1 ; 0$ & $4 ; 3.689$ & $1 ; 0$ & -3.903 \\
\hline 14 & $4 ; 2.773$ & $1 ; 0.693$ & $2 ;-0.693$ & $3 ;-1.609$ & $4 ; 0.470$ & $1 ; 2.708$ & $2 ; 0.693$ & $3 ; 3.401$ & $4 ; 2.079$ & -3.001 \\
\hline 15 & $1 ; 1.386$ & $2 ; 1.386$ & $2 ;-0.693$ & $3 ;-1.609$ & $3 ; 0.405$ & $4 ; 3.401$ & $4 ; 1.386$ & $1 ; 2.303$ & $1 ; 0$ & -3.014 \\
\hline
\end{tabular}

Bring the data in the above table into the Eq. (41) to obtain the contradiction equation where $\delta_{i}$ tends to zero. Solving the above contradictory equation by least squares method can obtain the following results:

$$
\begin{aligned}
& \vec{\beta}_{9}= {[-10.6544,0.1532,0.5418,-0.9747,-0.1512,} \\
&0.5415,1.0053,0.0195,0.5736,0.3079]^{\mathrm{T}} \\
& R_{2}= 2.3597 \times 10^{-5} v_{s}^{0.1532} P^{0.5418} v_{w}^{-0.9747} F_{t}^{-0.1512} \\
& \cdot G M^{0.5415} H^{1.0053} C_{c}^{0.0195} f^{0.5736} N^{0.3079}
\end{aligned}
$$

Based on the simulation data, the prediction model of $R_{2}$ is obtained. Then, the surface removal models of the other two stages can be obtained according to the Eq. (13). The next work of this paper is to verify the experimental model.

\section{EXPERIMENT AND ANALYSIS}

The experiment of this paper is completed on the robot system. The experimental system includes the robot manipulator, the clamping system, the blade workpiece, the grinding tool carrier (in this paper, the abrasive belt), the program for controlling the machining path, and the program. Auxiliary equipment 
such as cooling system, force sensing and control system, surface quality inspection system, etc.

Fig. 16 is a general overview of the robotic gripping blade. This image shows the robot arm holding the blade. One side of the blade is in contact with the contact wheel of the belt machine. The belt machine is specifically designed for the surface machining of the blade. Fig. 17 is a physical diagram of the connection of the tail tenon and the mechanical arm of the blade. The caliper in the figure is specially designed for the shape of the compressor tail stern of a certain type, combined with the rigidity requirement of the mechanical arm, the clamping condition is stable but its weight is not big.

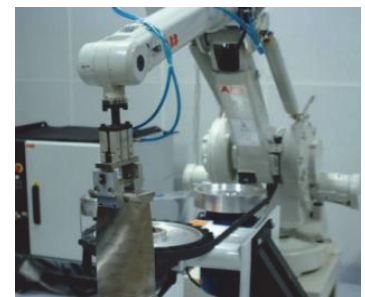

Figure 16 General overview of robot clamping blades

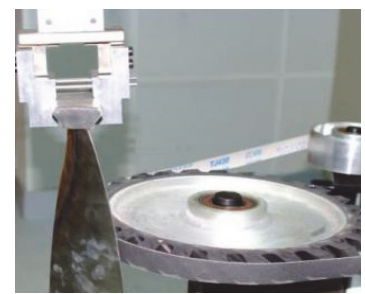

Figure 17 Blade tail and mechanical arm connection

Fig. 18 and Fig. 19 are partial enlarged views of the blade and the belt contact wheel. If the traces on the surface of the blade after processing are carefully observed, the regular fish scales can be seen with the naked eye, and the ripples have no significant surface roughness distribution. Fig. 19 is a view showing a case where the blade angle tends to the side surface, and the blade surface after polishing with a high mesh belt has a slight mirror effect, of course, the mirror effect is relatively low level, whose performance to the compressor is not too significant to get a large obvious effect.

The purpose of our experiment is to verify that the conclusions obtained in Chapter 4 are correct. In other words, we need to verify that this conclusion (which is the surface parametric model derived from the simulation) predicts the actual processing. Due to the huge complexity of the processing experiment, the blade material is too expensive. In this paper, five sets of processing experiments under different conditions were recorded. The condition parameters and measurement results are shown in Tab. 3 .

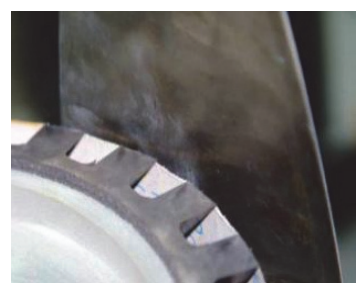

Figure 18 Partial enlarged view

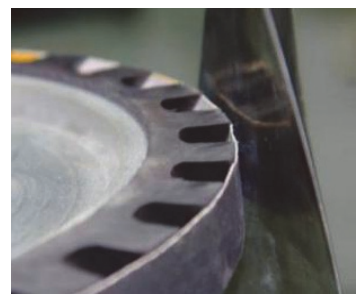

Figure 19 Observation tends to the side

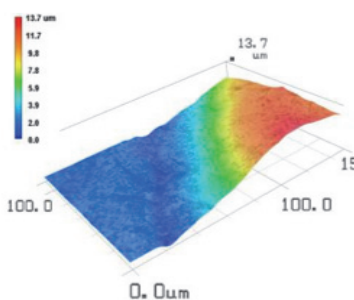

(a)

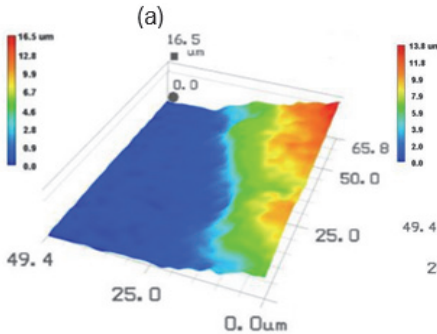

(c)

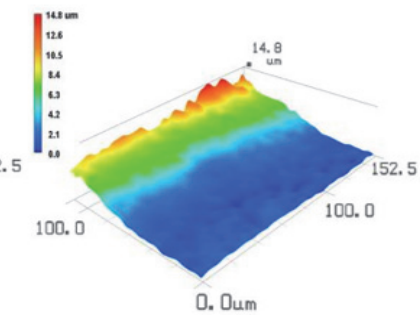

(b)

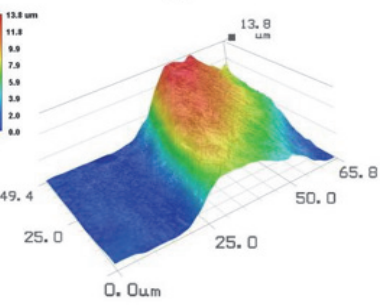

(d)

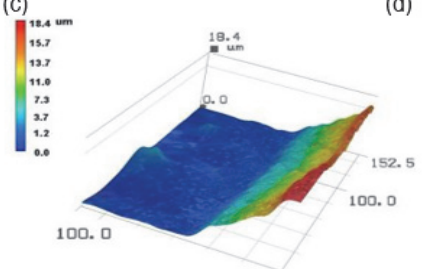

Figure 20 Measurement result of the surface after the experiment

Table 3 Condition parameters and measurement results

\begin{tabular}{|c|c|c|c|c|c|c|c|c|c|c|c|}
\hline Factor & $v_{\mathrm{s}} / \mathrm{m} / \mathrm{s}$ & $P / \mathrm{N}$ & $v_{w} / \mathrm{m} / \mathrm{min}$ & $F_{t} / \mathrm{N}$ & $G_{m}$ & $H / \mathrm{HA}$ & $C_{c} / \mathrm{kg} / \mathrm{min}$ & $N$ & $f$ & $R_{M}$ & $R_{T}$ \\
\hline 1 & 4.0 & 2.0 & 0.3 & 0.1 & 1.1 & 15.0 & 1.0 & 10 & 1 & 14.7 & 13.7 \\
\hline 2 & 8.0 & 2.0 & 0.7 & 0.1 & 1.1 & 15.0 & 1.0 & 10 & 2 & 16.9 & 14.8 \\
\hline 3 & 8.0 & 4.0 & 0.5 & 0.1 & 1.1 & 15.0 & 1.0 & 10 & 2 & 15.4 & 16.5 \\
\hline 4 & 12.0 & 4.0 & 0.7 & 0.1 & 1.1 & 15.0 & 2.0 & 10 & 2 & 14.9 & 13.8 \\
\hline 5 & 12.0 & 6.0 & 0.5 & 0.1 & 1.1 & 15.0 & 2.0 & 10 & 2 & 16.1 & 18.4 \\
\hline
\end{tabular}

Fig. 20a to Fig. 20e are the measurement results of the experimental groups 1-5 respectively. As shown in the figure, in order to facilitate the intuitive expression of the measurement results, the measurement area selects the boundary between the processed area and the unprocessed area, and the appropriate measurement multiple is selected 
to make the processed area and the unprocessed area in one field of view, the height difference between the two can represent the amount of removal produced by this set of experiments, which are marked in the picture.

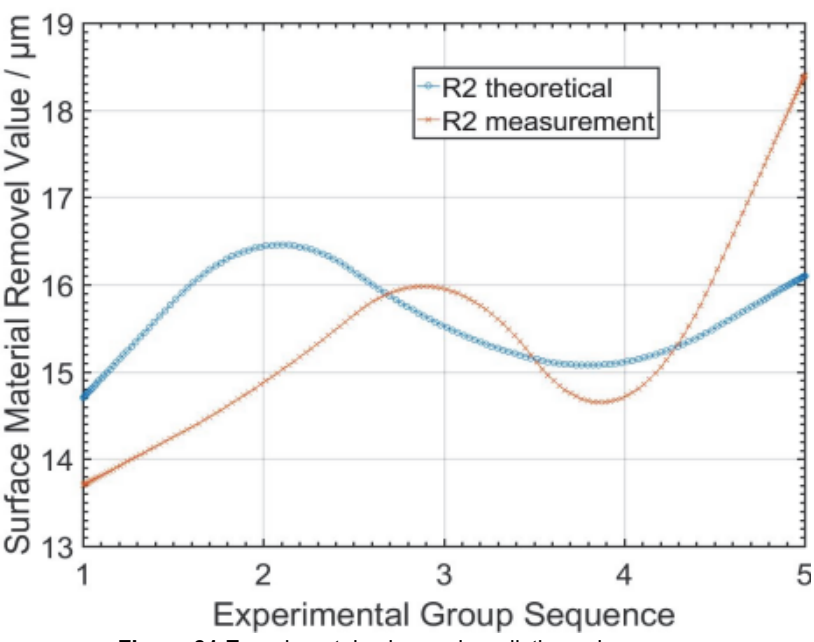

Figure 21 Experimental value and predictive value curve

The experimental measurements of the surface removal amount, and the predictive calculations of the corresponding experimental group, are plotted in Fig. 21. From this figure, it can be roughly judged that although there is a certain amount of deviation between the two sets of data, their increase or decrease is consistent, in other words, this parametric prediction model is predictive. The predicted and measured values are analyzed and plotted in Fig. 22. The maximum absolute error is $-2.4 \mu \mathrm{m}$, the minimum absolute error is $1.02 \mu \mathrm{m}$, the maximum relative error is $14 \%$, and the minimum relative error is $7 \%$.

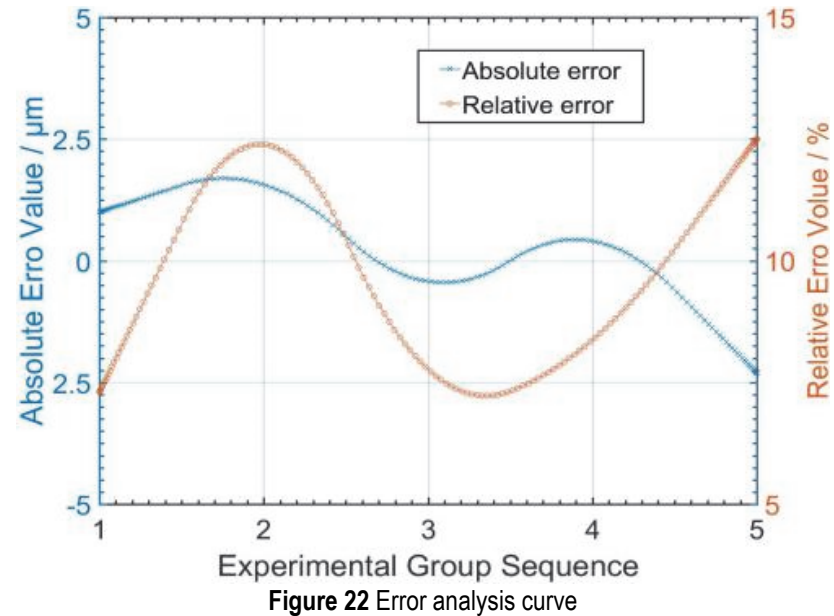

\section{CONCLUSIONS AND PROSPECTS}

In this paper, the abrasive state of the grinding tool is divided into initial wear stage, stable wear stage and sharp wear stage. Based on this, a parametric prediction model of engine blade surface material removal is established. In the simulation of blade surface machining, the blade is divided into several sections according to the direction from the blade root to the tip of the blade, and the curve fitting of the outer contour becomes more convenient to reduce the calculation amount. Through a series of simulation calculations, the expressions of the above parametric prediction models are obtained, and several sets of experiments are carried out to verify the feasibility of this prediction model. Obviously, the design, manufacture and assembly methods of engine blades will be the hotspot of future research. There are many research topics around the blades. Different companies, schools and researchers have proposed various methods, which not only promote the aviation industry, but also reduce operating costs in the aviation industry.

\section{REFERENCES}

[1] Peetermans, S. \& Lehmann, E. H. (2016). Simultaneous Neutron Transmission and Diffraction Imaging Investigation of Single Crystal Nickel-based Superalloy Turbine Blades. Ndt\& E International, 79, 109-113.

[2] Wang, R., Jiang, K., et al. (2016). Thermomechanical Fatigue Failure Investigation on A Single Crystal Nickel Superalloy Turbine Blade. Engineering Failure Analysis, 66, 284-295.

[3] Mavromihales, M., Mason, J., \& Weston, W. (2003). A Case of Reverse Engineering for The Manufacture of Wide Chord Fan Blades (WCFB) Used in Rolls Royce Aero Engines. Journal of Materials Processing Technology, 134(3), 279286.

[4] Qiu, L., Zeng, X., Ji, S. M., et al. (2018). Research on Combustion Chamber Structure Improvement and Ignition System of Missile Turbojet Engine for Diesel Fuel. Tehnicki Vejsnik-Technical Gazette, 25(6), 1792-1800.

[5] Na, Y. S., Yeom, J. T., et al. (2000). Simulation of Microstructures For Alloy 718 Blade Forging Using 3D FEM Simulator. Journal of Wuhan University of Hydraulic \& Electric Engineering, 141(3),337-342.

[6] Huang, W., Huang, C. F., et al. (2009). Key Manufacturing Technology Research of Advanced Aero-engine. Defense Manufacturing Technology, 03, 42-52.

[7] Takeuchi, Y. \& Watanabe, T. (1992). Generation of 5-axis Control Collision-free Tool Path and Post Processing for NC Data. International Institution for Production Engineering Research Annals, 41(1), 539-542.

[8] Cheng, H. Y. \& She, C. H. (2000). Studies on the Combination of the Forward and Reverse Postprocessor for Multi-axis Machine Tools. Proceeding of the Institution of Mechanical Engineers, Part B, 32(5), 77-87.

[9] She, C. H. \& Chang, C. C. (2007). Design of A Generic Fiveaxis Postprocessor Based On Generalized Kinematics Model of Machine Tool. International Journal of Machine Tools And Manufacture, 47(3), 537-545.

[10] Lai, X. D., Zhang, Q. H., et al. (2009). Digital Manufacture of Large-grade Hydro Turbine"s blades. Journal of Materials Processing Tech, 209(11), 4963-4969.

[11] Ho, S., Sarma, S., Adachi, Y. (2001). Real-time Interference Analysis Between A Tool and An Environment. Computer Aided Design, 33, 935-947.

[12] Julien, C. J., Gerard, P., Emmanuel, D. (2009). New Approach To 5-axis Flank Milling of Free-form Surfaces: Computation of Adapted Tool Shape. Computer Aided Design. 41(12), 918-929.

[13] Mejia, U. M., Dominguez, G. A., et al. (2016). Triangulation Intersection Approach From Poisson's Equation Applied To Automatic Tool Selection In Computer Numerical Control Mill-lathe. Proceedings of the Institution of Mechanical Engineers, Part B: Journal of Engineering Manufacture, 230(4), 722-731.

[14] Safka, J., Lachman, M., et al. (2015). Optimal Tool Path Searching and Tool Selection For Machining of Complex Surfaces. International Workshop of Electronics, Control, Measurement, Signals and Their Application to Mechatronics, 1-4. 
[15] Zeng, X., Ji, S. M., Jin, M. S., Tan, D. P., \& Ge, J. Q. (2016). Research on Dynamic Characteristic of Softness Consolidation Abrasives in Machining Process. The International Journal of Advanced Manufacturing Technology, 82(5-8), 1115-1125.

\section{Contact information:}

\section{Lei QIU}

Ningbo University of Technology,

Ningbo 305211, P. R. China,

Zhejiang University of Technology,

Hangzhou 310032, P. R. China

\section{Shiming J}

(Corresponding author)

Zhejiang University of Technology,

Hangzhou 310032, P. R. China.

Key Laboratory of E\&M,

Ministry of Education \& Zhejiang Province, Hangzhou 310032, P. R. China

E-mail: jishiming_zjut@163.com

\section{Xi ZENG}

Zhejiang University of Technology,

Hangzhou 310032, P. R. China,

The State Key Lab of Fluid Power and Mechatronic Systems,

Zhejiang university, Hangzhou 310058, P. R. China

\section{Dapeng TAN}

Key Laboratory of E\&M,

Ministry of Education \& Zhejiang Province,

Hangzhou 310032, P. R. China

\section{Man GE}

Key Laboratory of E\&M,

Ministry of Education \& Zhejiang Province,

Hangzhou 310032, P. R. China 